\title{
El camino suave del agua. Una alternativa para superar la crisis en la zona conurbada de Zacatecas y Guadalupe
}

\author{
Darcy Tetreault \\ Cindy McCulligh
}

Ubicada en el centro-norte de México, la Zona Conurbada de Zacatecas y Guadalupe (ZCZG) enfrenta una crisis del agua. Se ubica en una región donde el clima es semiseco, templado y árido, con sólo alrededor de $450 \mathrm{~mm}$ anuales de precipitación. Las aguas superficiales son escasas, y por tanto, se explotan los acuiferos para abastecer las necesidades de la población urbana. La región circundante depende de los mismos acuíferos para la irrigación de cultivos, con un bajo nivel de tecnificación. Además, hay industrias en la región - sobre todo la cervecería- que consumen grandes cantidades de agua en la producción de bienes para los mercados nacional e internacional. Con todo, los tres acuiferos que abastecen la zCZG (Benito Juárez, Calera y Guadalupe Bañuelos) están sobreexplotados. lo aue amenaza la sustentabilidad de la ciudad v las actividades productivas locales.

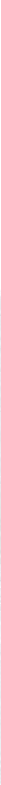

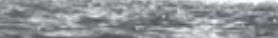


S egún la Comisión Nacional del Agua (Conagua), el acuífero Benito Juárez tiene un déficit de 1.14 millones de metros cúbicos por año ( $\mathrm{mm}^{3}$ /año); el acuífero Calera, de $67.75 \mathrm{~mm}^{3} /$ año, y el acuífero Guadalupe Bañuelos, de $1.95 \mathrm{~mm}^{3}$ / año. ${ }^{1}$ Ahora bien, es importante señalar que existe cierta incertidumbre con respecto a estos cálculos porque se basan en las concesiones, no en la cantidad real de agua que se extrae de los acuíferos. Hay un número desconocido de pozos clandestinos, y entre $25 \%$ y $50 \%$ de los pozos registrados no tienen medidores. ${ }^{2}$ En todo caso, se puede afirmar que los tres acuíferos están sobreexplotados debido al «descenso acelerado y progresivo de los niveles del agua subterránea». ${ }^{3}$

Basado en los datos de Conagua, $72.2 \%$ del agua extraída de los tres acuíferos se destina al sector agrícola, 20.9\% al sector público urbano y $6.5 \%$ al sector industrial. ${ }^{4}$ Así, cualquier solución integral para enfrentar la crisis del agua en la Zona Conurbada ZacatecasGuadalupe (zCZG) tiene que prestar mucha atención al sector agrícola a fin de reducir la demanda de agua subterránea, sin socavar las bases de la economía campesina. Aunque la zona urbana consume menos agua, no quiere decir que los esfuerzos para hacer este consumo más eficiente sean menos importantes. Todo lo contrario. La cantidad de agua destinada al consumo urbano de por sí justifica medidas para cuidarla. Además, tomando en cuenta la concentración demográfica, los vínculos familiares entre el campo y la ciudad y la centralización del poder político a nivel estatal, la ZCZG representa un espacio oportuno para concientizar a la población en general sobre la problemática del agua y para fomentar una nueva cultura en torno al líquido vital.

\section{El MANEJO DEL AGUA EN LA ZCZG}

Si bien los tres niveles de gobierno inciden en la gestión del agua en la ZCZG, lo cierto es que la principal responsabilidad para pro- veer agua entubada corresponde a la Junta Intermunicipal de Agua Potable y Alcantarillado de Zacatecas (JIAPAZ). Este organismo público descentralizado maneja el agua en los municipios de Zacatecas, Guadalupe, Morelos y Vetagrande. Su servicio tiene amplia cobertura. Según el Instituto Nacional de Estadística y Geografía (INEGI), 98.7\% de las viviendas en los cuatro municipios atendidos por dicho organismo cuenta con agua entubada. ${ }^{5}$ No obstante, el servicio no es constante; es muy común que se racione el agua por escasez, principalmente en primavera y verano.

La red de captación y distribución de agua de la mancha urbana se compone de cuatro principales sistemas: la Zacatecana, que empezó a extraer aguas del subsuelo en 1938; el sistema Calera, también conocido como La Joya, que data de 1966; el sistema Guadalupe Bañuelos (llamado alternativamente Bañuelos-San Ramón), que inició sus actividades en 1982, y el más reciente, Benito Juárez, que surgió en 1991. Además, existen tres subsistemas: Morelos, que forma parte del sistema Calera; Hormigueros, que se integra en el sistema Bañuelos-San Ramón, y Pimienta, creado en 2000-2001, con tres pozos en el sistema Benito Juárez y uno en el sistema Calera. ${ }^{6}$ En conjunto, la red de captación se compone de 52 pozos y abastece un caudal de 825 litros por segundo.

Tomando en cuenta que la población total de los cuatro municipios mencionados es de 308,821 habitantes, se puede calcular que el consumo en promedio es de 230.8 litros diarios de agua por habitante $(1 / \mathrm{d} / \mathrm{h})$. Esta cifra es considerablemente más alta que el estándar internacional de $150 \mathrm{l} / \mathrm{d} / \mathrm{h}$ para las ciudades sustentables, ${ }^{7}$ y si se compara con el uso de agua en algunas ciudades europeas, donde el consumo per cápita es alrededor de 100 litros diarios, se pone en evidencia el amplio margen que existe para mejorar la eficiencia del uso de agua en la ZCZG.

¿Por qué se consume tanta agua en esta zona urbana? Para empezar, se puede señalar el problema de las fugas. Se estima que un poco más de $40 \%$ del agua manejada por JIAPAZ se pierde en fugas, ya sea por el deterioro de la red de distribución o por tomas clandestinas. ${ }^{8}$ Otra razón por la cual el consumo de agua per cápita resulta excesivo es que hay pocos incentivos monetarios para que los usuarios aprovechen de manera eficiente el líquido vital o, inversamente, desincentivos para evitar el derroche. El sistema tarifario de JIAPAZ contempla tres zonas socioeconómicas: la primera es para fraccionamientos marginados, la segunda - la más común- es para colonias medias y la tercera es para las colonias con bienes raíces de alto valor. Si bien las tarifas existentes están escalonadas en cada una de estas zonas, el consumo mínimo de 10 metros cúbicos al mes $\left(\mathrm{m}^{3} / \mathrm{mes}\right)$ deja cierto margen para que las familias zacatecanas y guadalupenses no tengan que preocuparse por ahorrar el agua. Si el consumo es menor a $10 \mathrm{~m}^{3} / \mathrm{mes}$, pagan lo mismo, sin importar la cantidad de agua consumida. Las familias que utilizan menos de 10 $\mathrm{m}^{3} /$ mes no sólo no tienen desincentivos para derrochar el agua, sino 
que al contrario: mantienen el incentivo monetario de acercarse al consumo mínimo que les cobran.

Más aún, hay miles de usuarios en la zCZG que tienen cero incentivo monetario para consumir el agua de manera eficiente. De un total de 92,714 usuarios, 11,280 tienen una «cuota fija», ${ }^{9}$ en otras palabras, no tienen medidor y, por tanto, pagan el precio del consumo mínimo sin importar la cantidad de agua que consuman. Además, según Conagua, ${ }^{10}$ hay 5182 usuarios en la ciudad de Zacatecas que tienen «medidores sin lectura o sin funcionar». En estos casos, también el usuario paga el precio del consumo mínimo, sin importar la cantidad real del consumo.

Cabe señalar que el 25 de octubre de 2008, en el contexto de una crisis financiera de JIAPAZ, se aumentaron las tarifas por primera vez desde 1999 y el consumo mínimo fue reducido de $15 \mathrm{~m}^{3} / \mathrm{mes}$ a $10 \mathrm{~m}^{3} / \mathrm{mes}$. Sin embargo, el organismo intermunicipal no informó a los usuarios con anticipación, es decir, no realizó una campaña de concientización para explicar los objetivos de los cambios tarifarios y, por tanto, provocó un rechazo generalizado. ${ }^{11}$ Cuatro meses después, con la intervención de la entonces gobernadora Amalia García Medina, la JiApaz tuvo que dar un paso hacia atrás al reducir las tarifas en $30 \%,{ }^{12}$ medida con la cual se fijaron los precios actuales a partir de un consumo mínimo de $10 \mathrm{~m}^{3} /$ mes.

\section{EL CAMINO SUAVE DEL AGUA}

La sobrexplotación y la contaminación de acuíferos y fuentes de agua superficiales, así como la creciente concentración de la población en centros urbanos implican grandes retos para la provisión de agua a ciudades a nivel global. Tanto en países desarrollados como en desarrollo, estos fenómenos se ven agravados por eventos de sequía y, al mismo tiempo, los escenarios de cambio climático prevén permutas en regímenes de precipitación que impulsan búsquedas de alternativas para enfrentar un futuro incierto. Cada vez más, esta búsqueda no sólo contempla opciones «tradicionales» que tratan de identificar nuevas fuentes de agua para aumentar el abastecimiento a través de grandes obras de infraestructura hidráulica (presas, acueductos, sistemas de pozos), sino que se integran opciones de conservación, eficiencia, reúso, captación de aguas pluviales y reclamación de aguas tratadas, según las posibilidades hidrológicas, técnicas y culturales que existen en cada sitio. Este abanico de opciones no sólo representa una gama de posibilidades técnicas sino que puede constituir lo que Gleik llama un cambio de «paradigma del agua» para incorporar valores ecológicos en las políticas sobre el agua, enfocarse en satisfacer necesidades humanas básicas y romper el vínculo entre crecimiento económico y uso del agua. ${ }^{13}$ Esto también se ha llamado el «camino suave para el agua» (soft path for water), donde lo importante es aumentar la productividad del agua y trabajar con las poblaciones locales para satisfacer las necesidades relacionadas con el agua, más que simplemente abastecer agua; identificar la calidad del agua necesaria para cada actividad, ya que no todas las actividades requieren de agua de calidad potable (inodoros, jardines); incorporar sistemas descentralizados e involucrar a usuarios y grupos comunitarios en la gestión del agua. ${ }^{14}$

En un estudio reciente acerca de la «autosuficiencia» del agua urbana, a partir del análisis de quince casos a nivel global, donde se implementa la captación de agua lluvia, el reciclaje del agua o la desalinización, Rygaard, Binning y Albrechtsen concluyen que mientras no exista una panacea para el estrés del agua urbana, «un sistema de agua diversificado es más flexible y seguro frente a cambios de políticas o la variabilidad natural, y puede ser que la panacea sea la diversificación». ${ }^{15}$ Esa diversificación puede incluir explorar algunas de las posibilidades mencionadas a continuación.

Conservar el agua y hacer más eficiente su uso en las ciudades tiene que ser parte de cualquier estrategia, especialmente en las zonas áridas y semiáridas. El nivel de consumo de agua en las ciudades varía mucho, desde ciudades muy eficientes como Berlín, donde es de $110 \mathrm{l} / \mathrm{d} / \mathrm{h},{ }^{16}$ hasta las ciudades de California, donde casi alcanza los 400 1/d/h. ${ }^{17}$ Reducir el consumo promedio del agua y acercarse a los niveles menores tiene que partir de saber dónde y cómo se consume el agua: uso residencial, comercial, industrial. En el ámbito residencial, focalizar las acciones de ahorro también debe basarse en saber las proporciones de uso dentro de las casas versus en los exteriores, como los jardines, y con más detalle dentro de las casas, para poder saber el porcentaje del agua que se destina a cada uso: inodoros, regaderas, grifos, fugas, lavado de ropa y otros usos.

Mientras los precios que desincentiven el derroche del agua son importantes, vale la pena subrayar que es esencial garantizar un mínimo de agua para todos los hogares sin importar su capacidad de pago. Arrojo sugiere que los primeros 30 a 40 litros por 
habitante por día sean abastecidos en forma gratuita, por lo menos para los hogares más pobres, para garantizar el derecho humano al agua. ${ }^{18}$ Hay que mencionar, además, que al consumir menos agua en los hogares y comercios no sólo se limita la extracción de los acuíferos, sino que también se generan ahorros en la energía requerida para bombear, potabilizar, distribuir, recolectar y sanear el agua, así como los insumos necesarios para la potabilización y el tratamiento.

Hablar de conservar el agua en relación con una zona urbana donde $40 \%$ del agua se pierde resulta algo ingenuo. La conservación del agua tendrá que contemplar la inversión necesaria para reducir el nivel de pérdidas físicas. Biswas hace hincapié en el ejemplo de Phnom Penh, capital de Camboya, para demostrar que no sólo en los países desarrollados se pueden reducir las pérdidas. ${ }^{19} \mathrm{En}$ Phnom Penh, en 1993 se perdía 83\% del agua debido a fugas y tomas clandestinas; este número se logró reducir a 35\% para 2000, y en 2009 fue sólo de 6\%. También se ha logrado entregar agua limpia a toda la población y darle viabilidad financiera al operador municipal. ${ }^{20}$ Para el caso de la ZCZG, sería importante el proceso de aumentar la medición del agua: sólo así se podrá acercarse a una imagen más clara de las pérdidas y niveles de consumo reales para implementar acciones de conservación.

Aparte de reducir el consumo a través de las estrategias mencionadas, en diversas partes del mundo se retoma y aplica de diferentes maneras la captación de aguas pluviales como fuente alterna de agua para usos potables y no potables. Tradicionalmente, la captación de agua de lluvia, practicada en zonas áridas y semiáridas, se realiza de dos manera principales: captación en techos para usos domésticos e incluso para consumo humano (posterior a su tratamiento) y captación en el terreno mediante la canalización de aguas a bordos o presas, lo que sirve además para mantener los niveles de aguas subterráneas. ${ }^{21}$ La captación de agua de lluvia se practica en gran escala en importantes ciudades de India, Singapur, Corea, Japón, Alemania, Estados Unidos y otros países. Puede aplicarse a nivel domiciliario o ser parte de proyectos que cubran zonas más grandes. Dependiendo del nivel de tratamiento de las aguas, puede usarse desde jardines hasta para el consumo humano.

El reúso de las aguas tratadas puede darse en las zonas urbanas y ciertas aplicaciones industriales, adicional a su uso en riego agrícola. Las aguas residuales tratadas son un recurso valioso; en Israel, $20 \%$ de la demanda del agua del país se satisface con aguas residuales reclamadas. En el caso de Singapur, la meta es que 30\% de sus necesidades de agua se surtan de aguas residuales reclamadas, con usos primordialmente en la industria. ${ }^{22}$

Las alternativas brevemente reseñadas no pretenden cubrir todas las opciones que existen, sino resaltar algunas de las más importantes. En todos estos casos, la participación ciudadana es un elemento esencial para que sean exitosos, tanto programas que buscan reducir el consumo del agua como los que fomentan la implementación de tecnologías de captación o reúso. Por un lado, la conservación parte de saber dónde y para qué la gente usa el agua, y satisfacer esas necesidades con el mínimo de agua posible. Por otra parte, la falta del involucramiento de la población puede generar rechazo a las alternativas propuestas. El papel esencial de la ciudadanía en proponer y apropiarse de las medidas descritas puede generar la desconfianza de los operadores de sistemas de aguas, tradicionalmente dominados por ingenieros, quienes pueden subestimar el potencial de los ahorros que dependen del comportamiento de los usuarios. ${ }^{23} \mathrm{Al}$ mismo tiempo, la participación necesaria va más allá de los tradicionales programas de cultura del agua, que se aplican en el país, muchas veces sin metas o mensajes claros. Walsh, por ejemplo, cuestiona de manera interesante el programa de cultura del agua aplicado en Matamoros, Tamaulipas, con pláticas en las escuelas que invitan a los niños a ser «detectives del agua» y delatar a sus papás o vecinos por el desperdicio del agua. ${ }^{24}$ En lugar de enfrentar a los niños contra los adultos de esta manera, Walsh propone promover «culturas políticas de gestión del agua participativas [...] para que la reducción del agua no sea (ni se perciba como) una imposición de gobiernos y empresas del agua». ${ }^{25}$ La cultura del agua tendrá que ser una de gestión participativa si se quiere conservar el agua, proteger los acuíferos y explorar el potencial de la captación de aguas pluviales y el reúso de las aguas tratadas. 
1 Estos datos vienen de una serie de informes denominados Actualización de la disponibilidad media anual de agua subterránea, disponibles en la página de Internet de la CONAGUA (http://www.cna.gob.mx/).

2 Entrevista con el ingeniero Rafael Guardado y el ingeniero José Parra Hernández, del Departamento de Aguas Subterráneas, Dirección Local Zacatecas de la CONAGUA, el 22 de agosto de 2012.

3 CONAGUA, Nota informativa relativa a las fuentes de abastecimiento de agua potable para la ciudad de Zacatecas y zona conurbada, Zacatecas, Conagua, Gerencia Estatal Zacatecas, Subgerencia de Ingeniería, 2005.

4 CONAGUA, Resumen del número de aprovechamientos y volumen concesionado por uso (m3), y por acuíferos, Zacatecas, Dirección Local Zacatecas, Coordinación del Área Técnica, Departamento de Aguas Subterráneas, 2010.

5 INEgi, Censo de Población y Vivienda 2010 Zacatecas, México, 2011.

6 Patricia Rivera, Las perspectivas del manejo del agua urbana en zacatecas, ponencia presentada en el Primer Congreso Red de Investigadores Sociales sobre Agua, el 18 y el 19 de marzo de 2010, Centro de Capacitación del Instituto Mexicano de Tecnología del Agua, 2010.

7 Itzcóatl Tonatiuh Bravo-Padilla y Fabiola Figueroa Neri (coordinadores), El Proyecto Arcediano y el abastecimiento de agua potable de la Zona Conurbada de Guadalajara. Análisis de la Universidad de Guadalajara, Guadalajara, Universidad de Guadalajara, 2006, p. 36.

8 Conagua, Proyecto de fortalecimiento integrado del agua en México. Determinación de los costos económico-ambientales por la sobreexplotación del acuifero Guadalupe-Bañuelos, en el estado de Zacatecas, Zacatecas, Conagua y Organización Meteorológica Mundial, 2011, p. 28.

9 Banco Mundial, Análisis del uso y manejo de los recursos hídricos en el Estado de Zacatecas, Washington, Banco Mundial, 2012.

10 conagua, Situación del Subsector Agua Potable, Alcantarillado y Saneamiento Edición 2010, México, 2010, p. 88.

11 Patricia Rivera, op cit.

12 Fátima Ibarra, «Reducirán 30\% las tarifas del agua potable en cuatro municipios», Periódico El Sol de Zacatecas, 2009, en «http://www.oem.com.mx/ elsoldezacatecas/notas/n1024550.htm>.

13 Peter Gleik, «The Changing Water Paradigm: A Look at Twenty-first Century Water Resources Development», Water International, volumen 25, número 1, marzo, 2000, pp. 127-138.
14 Gary Wolff y Peter Gleik, «The Soft Path for Water» en Peter Gleik (editor), The World's Water 2002-2003: The Biennial Report on Freshwater Resources, Washington, Island Press, 2002.

15 Martin Rygaard, Philip J. Binning y Hans-Jørgen Albrechtsen, «Increasing urban water selfsufficiency: New era, new challenges», Journal of Environmental Management, volumen 92, 2011, p. 192.

16 Ibidem, p. 186.

17 Ryan Cahill y Jay Lund, Residential Water Conservation in Australia and California, Department of Civil and Environmental Engineering, University of California, Davis, 14 abril, 2011, p. 3.

18 Pedro Arrojo, «El reto ético de la crisis global del agua», Relaciones Internacionales, número 12, octubre, 2009, pp. 33-53.

19 Asit Biswas, «Water for a Thirsty Urban World», Brown Journal of World Affairs, vol. XVII, número I, otoño/invierno, 2010, pp. 147-162.

\section{Ibidem.}

21 Dheeraj Mandloi, Deepak Khare y Teena Pareek, «Rain water harvesting in Indore City: A demanding need for sustainable development», Journal of Chemical, Biological and Physical Sciences, volume 1, número 1, 2011, pp. 88-101.

22 Ryan Cahill y Jay Lund, Residential Water Conservation in Australia and California, p. 189.

23 Gary Wolff y Peter Gleik, «The Soft Path for Water», p. 9.

24 Casey Walsh, «Managing Urban Water Demand in Neoliberal Northern Mexico», Human Organization, volumen 70, número 1, primavera, 2011, pp. 54-62.

25 Ibidem, p. 61. 